\title{
OVOL1/2: drivers of epithelial differentiation in development, disease and reprogramming
}

Kritika Saxena ${ }^{1}$, Syamanthak Srikrishnan², Toni Celia-Terrassa ${ }^{3}$, Mohit Kumar Jolly ${ }^{1}$

${ }^{1}$ Centre for BioSystems Science and Engineering, Indian Institute of Science, Bangalore 560012 , India

2 Department of Biotechnology, Indian Institute of Technology, Kharagpur, 721302, India

${ }^{3}$ Cancer Research Program, IMIM (Hospital del Mar Medical Research Institute), 08003 Barcelona, Spain

\section{Short title: OVOL1/2 induce MET}

Corresponding Author: Dr. Mohit Kumar Jolly

Full name- Dr. Mohit Kumar Jolly

Department- BioSystems Science and Engineering (BSSE)

Institute- Indian Institute of Science (IISc), Bangalore, India

Postal address- C-wing, 3rd floor, Biological Sciences Building,

Indian Institute of Science,

Bengaluru, India 560012

Tel: $+91-80-22932751$

E-mail: mkjolly@iisc.ac.in

Number of Tables: 2

Number of Figures: 3

Word count: 3590

Keywords: OVOL1, OVOL2, Epithelial-Mesenchymal Transition, Mesenchymal-Epithelial Transition, Epithelial differentiation 
OVOL proteins (OVOL1 and OVOL2), vertebrate homologs of Drosophila OVO, are critical regulators of epithelial lineage determination and differentiation during embryonic development in tissues such as kidney, skin, mammary epithelia, testis. OVOL can inhibit Epithelial-Mesenchymal Transition (EMT) and/or can promote Mesenchymal-Epithelial Transition (MET). Moreover, they can regulate the stemness of cancer cells, thus playing an important role during cancer cell metastasis. Due to their central role in differentiation and maintenance of epithelial lineage, OVOL overexpression has been shown to be capable of reprogramming fibroblasts to epithelial cells. Here, we review the roles of OVOL mediated epithelial differentiation across multiple contexts - embryonic development, cancer progression, and cellular reprogramming.

\section{Introduction}

OVOL1 and OVOL2 are crucial transcription factors of epithelial lineages during vertebrate embryonic development and are involved in the maintenance of an epithelial state and terminal differentiation during tissue homeostasis (Fig. 1) [MacKay et al., 2006; Teng et al., 2007; Nair et al., 2006; Kohn et al., 2014; Sun et al., 2019]. OVOL1/2 can inhibit epithelial-mesenchymal transition (EMT) by directly repressing EMT inducing transcription factors (EMT-TFs) such as ZEB1, ZEB2, TWIST and promote the reverse of EMT - mesenchymal-epithelial transition (MET) by inducing the expression of cell-cell adhesion molecule E-cadherin [Kitazawa et al., 2019; Watanabe et al., 2019; Aue et al., 2015]. Thus, OVOL1/2 can be thought of as one of MET-inducing transcription factors (MET-TFs), similar to GRHL2 [Frisch et al., 2017; Mooney et al., 2017]. However, specific targets and functions of OVOL1 and OVOL2 are relatively poorly understood. A deeper appreciation of how OVOL1 and OVOL2 regulate epithelial differentiation and inhibit EMT will be required in the context of cancer metastasis, which remains to be the leading cause of cancer related deaths worldwide.

\section{OVOL proteins}

OVO is a nuclear protein expressed specifically in the female germline, and is critical for oogenesis and sex differentiation in Drosophila melanogaster [Mével-Ninio et al., 1995; Chidambaram et al., 1997; Oliver et al., 1987; Garfinkel et al., 1992]. ovo shares most of its coding sequence with shavenbaby ( $s v b)$ which is involved in epidermal morphogenesis [Payre et al., 1999; Mével-Ninio et al., 1995]. ovo/svb forms a complex gene locus with separate control regions performing two genetic functions: sexual differentiation and epidermal differentiation [Garfinkel et al., 1992]. OVO/SVB triggers F-actin redistribution that initiates cytoskeleton remodelling, thus functioning as an important regulator of epidermal differentiation [Delon et al., 2003]. The carboxyl terminal containing zinc finger domain (tetrad of $\mathrm{C} 2-\mathrm{H} 2$ ) of OVO is known to be evolutionary conserved from metazoans to vertebrates. Addition of various non-conserved sequences to primarily the $\mathrm{N}$-terminus region of the gene gave rise to different OVO-like (OVOL) genes during the course of evolution [Kumar et al., 2012].

Vertebrates have 3 ovol genes (ovol 1-3), among which ovol1 and ovol2 are relatively better studied (Table 1). Functional studies in model organisms have shown ovol genes to be 
involved in the development and differentiation of a number of epithelial lineages such as the skin, kidney and mammary epithelia [Johnson et al., 2001; Mével-Ninio et al., 1991; Oliver et al., 1987; Dai et al., 1998; Nair et al., 2007; Payre et al., 1999; MacKay et al., 2006]. OVOL1, the first OVO-like protein identified in mouse, was shown to be expressed in epidermis (skin, hair follicles and interfollicular epidermis), kidney and male germinal epithelia [Dai et al., 1998]. ovol1-deficient mouse displayed aberrant hair patterning, cystic kidneys and defective spermatogenesis [Li, 2005; Dai et al., 1998]. OVOL1 has been shown to function downstream of WNT/ß-catenin/LEF1 pathway in differentiating epidermal cells and hair follicles [Li et al., 2002b], and downstream of TGF-B/BMP-7/SMAD4 signalling pathway in keratinocytes [Kowanetz et al., 2004]. OVOL1 transcriptionally repressed its target genes such as $c-m y c$ and zeb1 by binding to their promoter at specific promoter sequence: CCGTTA, a recognition sequence that is also present in the ovol1 promoter, indicating its possible self-inhibition [Tsuji et al., 2018].

Similar to OVOL1, OVOL2 has been shown to be crucial for mouse embryonic development and ovol2 mutants show embryonic lethality [Unezaki et al., 2007; MacKay et al., 2006]. It is expressed during early-mid embryogenesis, particularly in the inner cell mass and in ectoderm derived tissues at later stages. OVOL2 expresses abundantly in the testis, however its expression has also been found in skin, stomach, intestine, ovary, heart and skeletal muscle [Li et al., 2002a].

Mouse ovol 2 consists of 5 exons, two of which are alternatively used to form spliced variants of OVOL2: OVOL2A (lacking $2^{\text {nd }}$ exon) and OVOL2B (lacking $1^{\text {st }}$ exon) [Li et al., 2002a]. The human ovol2 gene with six exons also have OVOL2A and OVOL2B transcript variants similar to mouse [Li et al., 2002a]. ovol2 null mutant mice showed expansion of neuroectoderm causing failure of the closure of cranial neural tube [MacKay et al., 2006]. Furthermore, defects in embryonic and extraembryonic vascularization along with improper heart development was also observed [Unezaki et al., 2007]. In addition, OVOL2 has been shown to function downstream of BMP signaling during neural/non-neural cell fate decision in chick embryos [Zhang et al., 2013]. Moreover, ovol2 is a downstream target of OVOL1: OVOL1 represses transcription of ovol 2 by directly binding to its promoter [Tsuji et al., 2018]. In addition, OVOL1 and OVOL2 recognize nearly identical DNA sequences for binding to their target genes (Table 2 ), suggesting possible regulation of one another and/or themselves [Wells et al., 2009; Lee et al., 2014].

\section{OVOL in embryonic development}

OVOL1 has been shown to regulate proliferation of epidermal cells during embryonic development in vivo using mouse models and in vitro using isolated keratinocytes from mouse [Nair et al., 2006]. OVOL1 was crucial for the terminal differentiation and restricting the proliferative potential of embryonic epidermal progenitor cells. In addition, cultured keratinocytes lacking OVOL1 failed to restrict their growth under growth inhibitory signals. ovol1-deficient suprabasal cells showed up-regulated c-MYC, which was shown to be transcriptionally repressed by OVOL1 by directly binding to its promoter, suggesting that OVOL 1 is required to repress c-myc for restricting cell proliferation [Nair et al., 2006].

OVOL2 has been shown to be capable of inducing mesendodermal fate in mouse development under the influence of BMP signaling - a known regulator of neural/non-neural 
cell fate decisions. BMP signaling inhibits neural differentiation and promotes differentiation of mesoderm, endoderm and epidermis [Zhang et al., 2013]. Thus, OVOL2 was found downregulated during neural differentiation of mouse embryonic stem cells (mESCs). Besides, inhibiting OVOL 2 in mESCs facilitated neural differentiation and inhibited mesendodermal differentiation. Moreover, in vivo experiments in chick embryos have shown BMP4 upregulates OVOL2 through SMAD1/5/8 which directly binds to the second intron of ovol2 and activates it [Zhang et al., 2013]. Ectopic expression of OVOL2 in the neural plate represses the expression of SOX2, a definitive marker of neural plate [Zhang et al., 2013]. Thus, OVOL2 seems to control proper germ layer development in multiple model organisms.

\section{2a) Epidermal/skin differentiation}

OVOL1 was first characterized functionally in mouse (gene: movo1) where it was shown to express in differentiating cells of epidermis and hair follicles, similar to its fly counterpart. It was found to be involved in hair and sperm formation as well, movo $1^{-/-}$mice produce aberrant hair and exhibit a limited ability to reproduce [Dai et al., 1998]. ovol1 mutant mice show perinatal lethality accompanied with epithelial cysts in kidney of embryonic onset and delayed acquisition of skin barrier [Teng et al., 2007]. Loss of OVOL1 was compensated by OVOL2 which was upregulated in ovol1-deficient epidermis. This effect can be mediated by direct repression of ovol2 by OVOL1 through two OVOL1 recognition sequence in its promoter (CCGTTA) [Teng et al., 2007]. ovol2-deficient mice display compromised wound healing characterized by aberrant epidermal cell migration and proliferation and defects in hair follicle matrix cell proliferation and differentiation [Hong et al., 2015].

The reciprocal regulation of epithelial and mesenchymal genes, as seen in EMT/MET, such as a mutually inhibitory feedback loop between OVOL1/2 and ZEB1 [Roca et al., 2013], is also implicated during the differentiation of embryonic ectoderm to neuro-ectoderm and surface ectoderm [Kitazawa et al., 2016]. Similar reciprocal inhibitory loops is a hallmark of multiple cell-fate decisions during embryonic development and disease [Zhou, and Huang, 2011; Sahoo et al., 2020]. While neuroectoderm derivatives expressed various mesenchymal genes, the corneal epithelial cells (CECs) arising from surface ectoderm have higher expression of epithelial genes such as cdh1, cldn1, krt3 and downregulated mesenchymal genes such as zeb1 and zeb2. OVOL2 is an important regulator of the human CEC transcriptional program [Kitazawa et al., 2016]. The OVOL1/OVOL2-ZEB1 axis seems to be relevant in epidermal progenitor cells too: ovol1/ovol2 conditional double knockout mice had blocked terminal differentiation and increased proliferation of embryonic epidermal progenitor cells with defects in a-catenin driven actin cytoskeleton reorganisation and adhesive maturation [Lee et al., 2014]. In addition, ovol1/ovol2-deficient epidermal cells displayed increased expression of cMYC and p63: transcription factors involved in stemness which were shown to be transcriptionally repressed by OVOL2 and exhibited changes similar to EMT which was reversed upon inhibition of EMT regulator ZEB1 [Lee et al., 2014]. Contrarily, another study has suggested the transient expansion of HaCaT keratinocytes upon OVOL2 depletion with a loss of long term proliferation potential and suppression of terminal differentiation [Wells et al., 2009], suggesting a potential context-dependent mediation of the functions of OVOL2.

Deletion of ZEB1 has also been shown to restore the directional migration of bulge hair follicle stem cells (Bu-HSCs) in ovol2-deficient mice which display characteristics of reduced proliferation and enhanced EMT [Haensel et al., 2019]. Overexpression of OVOL2 in mice 
epidermal basal cells disrupts cytoskeleton structure and display defective basal keratin network and defects in their association with hemidesmosomes, adhesion structures which anchors keratin filaments to cell/ basement membrane, resulting into skin blistering [Lee et al., 2017]. Put together, these studies suggest that epidermal differentiation program mediated by OVOL1 and/or OVOL2 may at least partly be mediated by their inhibition of EMT drivers such as ZEB1.

\section{2b) Mammary morphogenesis/Renal morphogenesis}

Another developmental scenario where the role of OVOL2 has been investigated is mammary and renal morphogenesis. Reminiscent of observations in epidermal differentiation, OVOL2 has been shown to be required for proper morphogenesis and regeneration of mammary epithelial by inhibiting EMT, as revealed through in vivo conditional knockout and lineage tracing experiments using mouse models. OVOL2 repressed EMT inducers such as ZEB1, ZEB2, TWIST and a TGF- $\beta$ mediated EMT was observed upon OVOL2 deletion [Watanabe et al., 2014]. Further, OVOL2 deletion blocked mammary ductal morphogenesis, depleted stem and progenitor cell population and induced EMT in epithelial cells to in in vivo mouse model. Phenotype associated with OVOL2 depletion was found to be reversible by inhibiting ZEB1 or TGF- $\beta$, further strengthening the importance of repressed EMT in ductal morphogenesis [Watanabe et al., 2014].

The GRHL2/OVOL2 pathway is involved in mouse renal epithelial morphogenesis through regulating lumen expansion and barrier formation in collecting duct epithelia by reinforcing the expression of CDH1, CLDN4 and RAB25. GRHL2 transactivated OVOL2 expression by associating with $\mathrm{H} 3$ lysine 4 trimethylation, thus functioning upstream of OVOL2. Moreover, OVOL2 induction was sufficient for the activation of CDH1, CLDN4 and RAB25, suggesting GRHL2 to be dispensable in the presence of OVOL2 [Aue et al., 2015].

\section{2c) Trophoblast development}

Human placental epithelium consists of cytotrophoblast which either proliferates to maintain sufficient reservoir of mononuclear progenitor cells or can differentiate that leads to the fusion of differentiated cells with the overlying syncytium forming syncytiotrophoblast [Renaud et al., 2015]. Syncytiotrophoblast forms the principal epithelial barrier between maternal and fetal blood and is important for nutrient, gas, waste and water exchange between the two blood circulations and synthesizes various hormones for fetal development and maintenance of pregnancy [Renaud et al., 2015]. OVOL1 has been shown to be robustly expressed in human placenta upon induction of trophoblast differentiation and is crucial for the differentiation of cytotrophoblast to syncytiotrophoblast that remains largely epithelial [Vićovac, and Aplin, 1996]. OVOL1 repressed genes involved in maintaining the progenitor cell state of cytotrophoblast such as myc, id1, tp63, and ascl2 which have OVOL1 recognition sequence (CCGTTA) within their proximal promoter regions. Deletion of OVOL1 abrogated cytotrophoblast fusion and inhibited genes involved in trophoblast cell fusion and hormonogenesis [Renaud et al., 2015]. Similarly, OVOL2 has been shown to be critical for the development of functional placenta in mice and OVOL2 depletion shows embryonic lethality. Mice placenta and trophoblast stem cells show higher expression of OVOL2 which is implicated in trophoblast differentiation. OVOL2 deletion inhibits differentiation of trophoblast stem cells such that they express higher levels of stem cell related genes such as eomes, 
esrrb, id2 and genes involved in differentiation such as gcm1, tpbpa, pr/3b1, syna are downregulated. In addition, ectopic expression of OVOL2 results into precocious differentiation of trophoblast stem cells [Jeyarajah et al., 2020]. Downregulation of OVOL2 has been implicated in EMT induction upon implantation of non-invasive bovine trophoblast to the uterine endometrial lining. In vitro experiments with cultured bovine trophoblast cells also showed similar induction of EMT only after attachment to endometrial epithelial layer via TEAD3/YAP signaling pathway. OVOL2 downregulation was paralleled with increase in expression of EMT transcription factors ZEB1 and SNAI2, EMT markers vimentin and Ncadherin and reduction in expression of epithelial marker E-cadherin [Bai et al., 2018]. This study highlights the criticality of balance between EMT (inhibited by OVOL2) and MET (promoted by OVOL2) during embryogenesis and development.

\section{OVOL and EMT in cancer}

EMT and MET are believed to be important for successful metastasis of cancer cells to distant locations. While EMT can trigger invasion and tumor cell dissemination, MET can facilitate conversion of mesenchymal carcinoma cells to their epithelial phenotype at distant sites where they can regain proliferation [Jolly et al., 2017]. OVOL1 and OVOL2 can induce MET in prostate and breast cancer cells characterized by changes in cell morphology and an increased expression of epithelial markers such as E-cadherin and ESRP1 and decreased levels of EMT inducers ZEB1, ZEB2 and SLUG (SNAI2) [Roca et al., 2013]. Importantly, ESRP1 and E-cadherin can also form mutually inhibitory loops with ZEB1, thus repressing EMT [Jolly et al., 2018b; Schmalhofer et al., 2009]. In addition, expression of OVOL1 and OVOL2 in mesenchymal prostate cancer cells decreased their metastatic potential in orthotopic mouse models of prostate cancer [Roca et al., 2013]. Similarly, OVOL2 has been shown to antagonize TGF- $\beta$ signaling and inhibited EMT during mammary tumor metastasis by repressing SMAD4 expression and interfering in SMAD4 and SMAD2/3 complex formation [Wu et al., 2017]. Thus, OVOL1/2-ZEB1 axis can form an important axis of regulation of EMT in cancer progression, as observed for Cancer Cell Line Encyclopedia (CCLE) cohort [Barretina et al., 2012] (Fig. 2).

OVOL1-OVOL2 axis has also been implicated in regulation of C-MYC in Bowen's disease and cutaneous squamous skin cancer. Bowen's disease, characterized by atypical keratinocytes, is a non-invasive variant form of squamous cell carcinoma (SCC) restricted to epidermis. It is characterized by higher expression of OVOL1 while C-MYC is downregulated. On the other hand, SCCs shows an opposite trend: OVOL1 is downregulated while c-MYC levels are higher. OVOL2 expression was downregulated in Bowen's disease and showed varied expression in squamous cell carcinoma: diffused in the cytoplasm with sporadic expression in nuclei. The expression pattern of OVOL1,OVOL2 and c-MYC in these two forms of skin deformities highlight the role of OVOL1-OVOL2 axis in restricting invasiveness of cell by modulating the expression of proto-oncogene c-MYC [lto et al., 2017]. Similar observation was made in actinic keratosis (AK) which is characterized by intraepidermal lesions and the progression of AK to cutaneous SCC (CSCC) is rare. AK display suppressed EMT owing to higher levels of OVOL1/OVOL2 and lower levels of ZEB1 and vimentin, contrary to higher levels of ZEB1 and vimentin in CSCC and lower levels of OVOL1/OVOL2_[Murata et al., 2020].

Furthermore, in A431 SCC cells, OVOL1 and OVOL2 knockdown increased the mRNA and protein levels of ZEB1. Also, OVOL2 knockdown increased the invasive capability of cells, 
suggesting OVOL2/ZEB1 crosstalk in modulating AK/cSCC progression [Murata et al., 2020]. OVOL2 mediated suppression of ZEB1 and promotion of MET is also reported in osteosarcoma [Liu et al., 2018]. A recent study has shown OVOL1 mediated suppression of proliferation, invasion and migration in oral squamous cell carcinoma (SCC-152) cells by inhibiting ZEB1 expression by directly binding to its promoter [Xu et al., 2019], further endorsing that OVOL2/ZEB1 feedback loop controls epithelial-mesenchymal plasticity across carcinomas.

Mathematical modeling of the feedback loop between ZEB1 and OVOL2 has revealed that in addition to epithelial (high OVOL2, low ZEB1) and mesenchymal (low OVOL2, high ZEB1), cells can acquire one or more hybrid epithelial/mesenchymal (medium OVOL2, medium ZEB1) states [Jolly et al., 2015; Jia et al., 2015; Hong et al., 2015] which can be the most plastic and aggressive state [Jolly et al., 2018a]. In H1975 lung cancer cells that can display a hybrid E/M stably over multiple passages, knockdown of OVOL2 increased the levels of mesenchymal markers, impaired collective cell migration and drove a complete EMT [Jolly et al., 2016], reminiscent of its role reported in mammary morphogenesis. In collective migration of terminal end buds during mammary development, and that of lung cancer cell lines, OVOL2 can be thought of as a "phenotypic stability factor" [Jolly et al., 2016] that can prevent "cells that have gained partial plasticity from crossing the line to undergo complete EMT" [Watanabe et al., 2014], thus acting as a gatekeeper of epithelial phenotype.

Similarly, in A549 cells, OVOL2 can suppress EMT by inhibiting the transcriptional activity of TWIST [Wang et al., 2017]. Low OVOL1 and high ZEB1 and FN1 expression was also seen in tumor buds of oral SCCs which form the invasive front and known to display EMT features [Jensen et al., 2015]. Low OVOL2 expression has been shown to be associated with worse overall survival in hepatocellular and colon carcinoma patients and correlated with EMT progression in patient HCC tissue samples [Fu et al., 2016; Ye et al., 2016]. However, a functional connection of OVOL with MET in vivo remains to be identified.

\section{OVOL, plasticity and stemness}

Hybrid E/M cells can facilitate collective cell migration as observed in clusters of circulating tumor cells (CTCs). Relative to individually migrating CTCs, CTC clusters tend to have increased survival of cancer cells in blood circulation [Saxena et al., 2020]. Moreover, in CTC clusters, the binding sites for stemness associated factors such as OCT4, NANOG and SOX2 are more hypomethylated [Gkountela et al., 2019]. Together, these factors may contribute to the disproportionately high metastatic propensity of CTC clusters [Aceto et al., 2014].

A mechanism based mathematical model coupling OVOL with EMT (miR-200/ZEB) and stemness (LIN28/let-7) circuit predicted that OVOL can not only stabilize a hybrid E/M phenotype, but also increase the stemness associated with hybrid E/M and/or epithelial phenotypes, while decreasing the stemness associated with mesenchymal state. Thus, OVOL can fine tune the positioning of "stemness window" on the "EMT axis" [Jolly et al., 2015]. Similarly, an increase in stemness by OVOL2 has been shown in nasopharyngeal cancer, where OVOL2 ectopic expression in mesenchymal cells could only induce partial epithelial character. In addition, inhibition of ZEB1 in ovol2-deficient cells decreased stemness without affecting their invasiveness, suggesting a crucial role of OVOL2 in stabilizing hybrid E/M phenotype and conferring stemness to it [Qi et al., 2018]. The transient receptor potential 
vanilloid 1 (TRPV1), a non-selective cation channel, has been shown to modulate the plasticity of hepatocyte. TRPV1 downregulation has been shown to be associated with activation of ZEB1 and inhibition of OVOL2 which promoted hepatocarcinogenesis in a SOX10-dependent manner [Xie et al., 2019]. Coordinated regulation of cell state by ZEB1-OVOL2-GRHL2 axis has been shown to be crucial for corneal endothelial cells and alteration in this axis results into posterior polymorphous corneal dystrophy (PPCD) characterized by aberrant activation of Wnt pathway, highlighting the role of ZEB1-OVOL2-GRHL2 axis in mediating cellular plasticity in corneal tissue homeostasis [Chung et al., 2019].

\section{OVOL and cellular reprogramming}

OVOLs are known to be critical regulators of epidermal fate determination and differentiation indicating their capability to confer epidermal phenotype to other cell types by reprogramming their gene expression [Kagawa et al., 2019]. OVOL1 in combination with some or all of the selected critical regulators of epidermal fate (PRDM1, p63, KLF4, ZNF750, and GRHL3) has been shown to be sufficient for the rapid conversion of human dermal fibroblasts to an induced keratinocyte phenotype (iKP) expressing keratinocyte specific genes: krt14 and gjb2 [Chen et al., 2014]. Furthermore, OVOL2 has been shown to cooperatively promote MET in fibroblasts to keratinocytes induced by epithelial lineage promoting transcription factors such as HNF1A, TP63, and KLF4 by inducing genes involved in epithelial phenotype and repressing fibroblastassociated genes [Watanabe et al., 2019]. Similarly, reprogramming of human fibroblast cells to corneal epithelial cells (CECs) was done by overexpressing core CEC network consisting of PAX6, OVOL2 and KLF4. In addition, OVOL2 was sufficient to directly reprogram neural ectoderm to a more epithelial cell state: surface ectoderm [Kitazawa et al., 2019].

\section{Conclusion}

OVOL (OVOL1/OVOL2) are critical regulators of normal epithelial differentiation as well as cancer cell differentiation (Fig. 3). They function downstream of growth signaling such as $W n t / \beta$-catenin and BMP-SMAD. They can inhibit EMT and promote MET in cancer, and have thus been implicated as prognostic marker for patient survival. OVOL1 and OVOL2 regulate many common targets, perform many redundant functions, and can compensate for each other, however, few targets and functions are specific to OVOL1 and OVOL2. Looking at the diverse roles of OVOL during embryonic development, cancer and reprogramming, it is important to identify and characterize upstream and downstream targets of OVOL and understand how various OVOL mediated pathways converge to perform similar functions.

\section{Statements}

Conflict of Interest Statement The authors have no conflicts of interest to declare.

Funding Sources This work is supported by Ramanujan Fellowship awarded by Science and Engineering Research Board (SERB), Department of Science and Technology (DST), Government of India (SB/S2/RJN-049/2018) to MKJ, and by the Instituto de Salud Carlos IIIFSE (MS17/00037) and the AECC lab grant awarded to TC-T.

Author Contributions KS prepared a first draft of the manuscript. KS, SS, TC-T and MKJ prepared figures and edited the manuscript. MKJ supervised the study. 


\section{References}

Aceto N, Bardia A, Miyamoto DT, Donaldson MC, Wittner BS, Spencer JA, et al.: Circulating Tumor Cell Clusters Are Oligoclonal Precursors of Breast Cancer Metastasis. Cell 2014;158:1110-1122.

Aue A, Hinze C, Walentin K, Ruffert J, Yurtdas Y, Werth M, et al.: A grainyhead-like 2/Ovolike 2 pathway regulates renal epithelial barrier function and lumen expansion. J Am Soc Nephrol 2015; DOI: 10.1681/ASN.2014080759

Bai R, Kusama K, Nakamura K, Sakurai T, Kimura K, Ideta A, et al.: Down-regulation of transcription factor OVOL2 contributes to epithelial-mesenchymal transition in a noninvasive type of trophoblast implantation to the maternal endometrium. FASEB J 2018; DOI: 10.1096/fj.201701131RR

Barretina J, Caponigro G, Stransky N, Venkatesan K, Margolin A a., Kim S, et al.: The Cancer Cell Line Encyclopedia enables predictive modelling of anticancer drug sensitivity. Nature 2012;483:603-607.

Chen Y, Mistry DS, Sen GL: Highly Rapid and Efficient Conversion of Human Fibroblasts to Keratinocyte-Like Cells. J Invest Dermatol 2014;134:335-344.

Chidambaram A, Allikmets R, Chandrasekarappa S, Guru SC, Modi W, Gerrard B, et al.: Characterization of a human homolog (OVOL1) of the Drosophila ovo gene, which maps to Chromosome 11q13. Mamm Genome 1997;8:950-951.

Chung DD, Zhang W, Jatavallabhula K, Barrington A, Jung JY, Aldave AJ: Alterations in GRHL2-OVOL2-ZEB1 axis and aberrant activation of Wnt signaling lead to altered gene transcription in posterior polymorphous corneal dystrophy. Exp Eye Res 2019; DOI: 10.1016/j.exer.2019.107696

Dai X, Schonbaum C, Degenstein L, Bai W, Mahowald A, Fuchs E: The ovo gene required for cuticle formation and oogenesis in flies is involved in hair formation and spermatogenesis in mice. Genes Dev 1998; DOI: 10.1101/gad.12.21.3452

Delon I, Chanut-Delalande H, Payre F: The Ovo/Shavenbaby transcription factor specifies actin remodelling during epidermal differentiation in Drosophila. Mech Dev 2003; DOI: 10.1016/S0925-4773(03)00081-9

Frisch SM, Farris JC, Pifer PM: Roles of Grainyhead-like transcription factors in cancer. Oncogene 2017;36:6067-6073.

Fu H, Qi L, Chen L, He Y, Zhang N, Guo H: Expression of Ovol2 is related to epithelial characteristics and shows a favorable clinical outcome in hepatocellular carcinoma. Onco Targets Ther 2016; DOI: 10.2147/OTT.S110409

Garfinkel MD, Lohe AR, Mahowald AP: Molecular genetics of the Drosophila melanogaster ovo locus, a gene required for sex determination of germline cells. Genetics 1992;

Gkountela S, Castro-Giner F, Szczerba BM, Vetter M, Landin J, Scherrer R, et al.: Circulating Tumor Cell Clustering Shapes DNA Methylation to Enable Metastasis Seeding. Cell 2019; DOI: 10.1016/j.cell.2018.11.046

Haensel D, Sun P, MacLean AL, Ma X, Zhou Y, Stemmler MP, et al.: An Ovol2-Zeb1 transcriptional circuit regulates epithelial directional migration and proliferation. EMBO Rep 2019;20. DOI: 10.15252/embr.201846273

Hong T, Watanabe K, Ta CH, Villarreal-Ponce A, Nie Q, Dai X: An Ovol2-Zeb1 Mutual Inhibitory Circuit Governs Bidirectional and Multi-step Transition between Epithelial and Mesenchymal States. PLoS Comput Biol 2015; DOI: 10.1371/journal.pcbi.1004569

Ito T, Tsuji G, Ohno F, Nakahara T, Uchi H, Furue M: Potential role of the OVOL1-OVOL2 axis and c-Myc in the progression of cutaneous squamous cell carcinoma. Mod Pathol 2017; DOI: $10.1038 /$ modpathol.2016.169

Jensen DH, Dabelsteen E, Specht L, Fiehn AMK, Therkildsen MH, Jønson L, et al.: Molecular profiling of tumour budding implicates TGF $\beta$-mediated epithelialmesenchymal transition as a therapeutic target in oral squamous cell carcinoma. $\mathrm{J}$ Pathol 2015; DOI: 10.1002/path.4550

Jeyarajah MJ, Jaju Bhattad G, Hillier DM, Renaud SJ: The Transcription Factor OVOL2 
Represses ID2 and Drives Differentiation of Trophoblast Stem Cells and Placental Development in Mice. Cells 2020;9:840.

Jia D, Jolly MK, Boareto M, Parsana P, Mooney SM, Pienta KJ, et al.: OVOL guides the epithelial-hybrid-mesenchymal transition. Oncotarget 2015; DOI:

10.18632/oncotarget.3623

Johnson AD, Fitzsimmons D, Hagman J, Chamberlin HM: EGL-38 Pax regulates the ovorelated gene lin-48 during Caenorhabditis elegans organ development. Development 2001;

Jolly MK, Jia D, Boareto M, Mani SA, Pienta KJ, Ben-Jacob E, et al.: Coupling the modules of EMT and stemness: A tunable 'stemness window' model. Oncotarget 2015;6:2516125174.

Jolly MK, Mani SA, Levine H: Hybrid epithelial/mesenchymal phenotype(s): The 'fittest' for metastasis? Biochim Biophys Acta - Rev Cancer 2018a;1870:151-157.

Jolly MK, Preca B-T, Tripathi SC, Jia D, George JT, Hanash SM, et al.: Interconnected feedback loops among ESRP1, HAS2, and CD44 regulate epithelial-mesenchymal plasticity in cancer. APL Bioeng 2018b; DOI: 10.1063/1.5024874

Jolly MK, Tripathi SC, Jia D, Mooney SM, Celiktas M, Hanash SM, et al.: Stability of the hybrid epithelial/mesenchymal phenotype. Oncotarget 2016;7. DOI:

10.18632/oncotarget.8166

Jolly MK, Ware KE, Gilja S, Somarelli JA, Levine H: EMT and MET: necessary or permissive for metastasis? Mol Oncol 2017;11:755-769.

Kagawa H, Shimamoto R, Kim S II, Oceguera-Yanez F, Yamamoto T, Schroeder T, et al.: OVOL1 Influences the Determination and Expansion of iPSC Reprogramming Intermediates. Stem Cell Reports 2019; DOI: 10.1016/j.stemcr.2018.12.008

Kitazawa K, Hikichi T, Nakamura T, Mitsunaga K, Tanaka A, Nakamura M, et al.: OVOL2 Maintains the Transcriptional Program of Human Corneal Epithelium by Suppressing Epithelial-to-Mesenchymal Transition. Cell Rep 2016;15:1359-1368.

Kitazawa K, Hikichi T, Nakamura T, Nakamura M, Sotozono C, Masui S, et al.: Direct Reprogramming Into Corneal Epithelial Cells Using a Transcriptional Network Comprising PAX6, OVOL2, and KLF4. Cornea 2019;38:S34-S41.

Kohn KW, Zeeberg BM, Reinhold WC, Pommier Y: Gene expression correlations in human cancer cell lines define molecular interaction networks for epithelial phenotype. PLoS One 2014; DOI: 10.1371/journal.pone.0099269

Kowanetz M, Valcourt U, Bergström R, Heldin C-H, Moustakas A: Id2 and Id3 Define the Potency of Cell Proliferation and Differentiation Responses to Transforming Growth Factor $\beta$ and Bone Morphogenetic Protein. Mol Cell Biol 2004;24:4241-4254.

Kumar A, Bhandari A, Sinha R, Sardar P, Sushma M, Goyal P, et al.: Molecular Phylogeny of OVOL Genes Illustrates a Conserved $\mathrm{C} 2 \mathrm{H} 2$ Zinc Finger Domain Coupled by Hypervariable Unstructured Regions. PLoS One 2012;7:e39399.

Lee B, Villarreal-Ponce A, Fallahi M, Ovadia J, Sun P, Yu Q-C, et al.: Transcriptional Mechanisms Link Epithelial Plasticity to Adhesion and Differentiation of Epidermal Progenitor Cells. Dev Cell 2014;29:47-58.

Lee B, Watanabe K, Haensel D, Sui JY, Dai X: Overexpression of Transcription Factor Ovol2 in Epidermal Progenitor Cells Results in Skin Blistering. J Invest Dermatol 2017; DOI: 10.1016/j.jid.2017.02.985

Li B: Ovol1 regulates meiotic pachytene progression during spermatogenesis by repressing Id2 expression. Development 2005;132:1463-1473.

Li B, Dai Q, Li L, Nair M, Mackay DR, Dai X: Ovol2, a mammalian homolog of Drosophila ovo: Gene structure, chromosomal mapping, and aberrant expression in blind-sterile mice. Genomics 2002a; DOI: 10.1006/geno.2002.6831

Li B, Mackay DR, Dai Q, Li TWH, Nair M, Fallahi M, et al.: The LEF1/ -catenin complex activates movo1, a mouse homolog of Drosophila ovo required for epidermal appendage differentiation. Proc Natl Acad Sci 2002b;99:6064-6069.

Liu J, Wu Q, Wang Y, Wei Y, Wu H, Duan L, et al.: Ovol2 induces mesenchymal\&ndash;epithelial transition via targeting ZEB1 in osteosarcoma. Onco 
Targets Ther 2018; Volume 11:2963-2973.

MacKay DR, Hu M, Li B, Rhéaume C, Dai X: The mouse Ovol2 gene is required for cranial neural tube development. Dev Biol 2006; DOI: 10.1016/j.ydbio.2005.12.003

Mével-Ninio M, Terracol R, Kafatos FC: The ovo gene of Drosophila encodes a zinc finger protein required for female germ line development. EMBO J 1991;10:2259-2266.

Mével-Ninio M, Terracol R, Salles C, Vincent A, Payre F: ovo, a Drosophila gene required for ovarian development, is specifically expressed in the germline and shares most of its coding sequences with shavenbaby, a gene involved in embryo patterning. Mech Dev 1995; DOI: 10.1016/0925-4773(94)00305-7

Mooney SM, Talebian V, Jolly MK, Jia D, Gromala M, Levine H, et al.: The GRHL2/ZEB Feedback Loop - A Key Axis in the Regulation of EMT in Breast Cancer. J Cell Biochem 2017; in press.

Murata M, Ito T, Tanaka Y, Yamamura K, Furue K, Furue M: OVOL2-Mediated ZEB1 Downregulation May Prevent Promotion of Actinic Keratosis to Cutaneous Squamous Cell Carcinoma. J Clin Med 2020; DOI: 10.3390/jcm9030618

Nair M, Bilanchone V, Ortt K, Sinha S, Dai X: Ovol1 represses its own transcription by competing with transcription activator $\mathrm{c}-\mathrm{Myb}$ and by recruiting histone deacetylase activity. Nucleic Acids Res 2007; DOI: 10.1093/nar/gkl1141

Nair M, Teng A, Bilanchone V, Agrawal A, Li B, Dai X: Ovol1 regulates the growth arrest of embryonic epidermal progenitor cells and represses c-myc transcription. J Cell Biol 2006; DOI: 10.1083/jcb.200508196

Oliver B, Perrimon N, Mahowald AP: The ovo locus is required for sex-specific germ line maintenance in Drosophila. Genes Dev 1987; DOI: 10.1101/gad.1.9.913

Payre F, Vincent A, Carreno S: ovo/svb Integrates Wingless and DER pathways to control epidermis differentiation. Nature 1999; DOI: 10.1038/22330

Qi XK, Han HQ, Zhang HJ, Xu M, Li L, Chen L, et al.: OVOL2 links stemness and metastasis via fine-tuning epithelial-mesenchymal transition in nasopharyngeal carcinoma. Theranostics 2018; DOI: 10.7150/thno.24003

Renaud SJ, Chakraborty D, Mason CW, Rumi MAK, Vivian JL, Soares MJ: OVO-like 1 regulates progenitor cell fate in human trophoblast development. Proc Natl Acad Sci 2015;112:E6175-E6184.

Roca H, Hernandez J, Weidner S, McEachin RC, Fuller D, Sud S, et al.: Transcription Factors OVOL1 and OVOL2 Induce the Mesenchymal to Epithelial Transition in Human Cancer. PLoS One 2013; DOI: 10.1371/journal.pone.0076773

Sahoo S, Singh D, Chakraborty P, Jolly MK: Emergent properties of the HNF4a-PPARg network may drive consequent phenotypic plasticity in NAFLD. J Clin Med 2020;9:870.

Saxena K, Jolly MK, Balamurugan K, Signaling D: Hypoxia, partial EMT and collective migration : emerging culprits in metastasis. Transl Oncol 2020;13:100845.

Schmalhofer O, Brabletz S, Brabletz T: E-cadherin, $\beta$-catenin, and ZEB1 in malignant progression of cancer. Cancer Metastasis Rev 2009;28:151-166.

Sun X, Li Z, Niu Y, Zhao L, Huang Y, Li Q, et al.: Jarid1b promotes epidermal differentiation by mediating the repression of Ship1 and activation of the AKT/Ovol1 pathway. Cell Prolif 2019;52. DOI: 10.1111/cpr.12638

Teng A, Nair M, Wells J, Segre JA, Dai X: Strain-dependent perinatal lethality of Ovol1deficient mice and identification of Ovol2 as a downstream target of Ovol1 in skin epidermis. Biochim Biophys Acta - Mol Basis Dis 2007;1772:89-95.

Tsuji G, Ito T, Chiba T, Mitoma C, Nakahara T, Uchi H, et al.: The role of the OVOL1OVOL2 axis in normal and diseased human skin. J Dermatol Sci 2018; DOI: 10.1016/j.jdermsci.2018.02.005

Unezaki S, Horai R, Sudo K, Iwakura Y, Ito S: Ovol2/Movo, a homologue of Drosophila ovo, is required for angiogenesis, heart formation and placental development in mice. Genes to Cells 2007;070606122915003-???

Vićovac L, Aplin JD: Epithelial-mesenchymal transition during trophoblast differentiation. Acta Anat 1996;156:202-16.

Wang Z-H, Li Z, Hu M, Yang Q-J, Yan S, Wu R-S, et al.: Ovol2 gene inhibits the Epithelial- 
to-Mesenchymal Transition in lung adenocarcinoma by transcriptionally repressing Twist1. Gene 2017;600:1-8.

Watanabe K, Liu Y, Noguchi S, Murray M, Chang JC, Kishima M, et al.: OVOL2 induces mesenchymal-to-epithelial transition in fibroblasts and enhances cell-state reprogramming towards epithelial lineages. Sci Rep 2019; DOI: 10.1038/s41598-01943021-z

Watanabe K, Villarreal-Ponce A, Sun P, Salmans ML, Fallahi M, Andersen B, et al.: Mammary morphogenesis and regeneration require the inhibition of EMT at terminal end buds by ovol2 transcriptional repressor. Dev Cell 2014; DOI: 10.1016/j.devcel.2014.03.006

Wells J, Lee B, Cai AQ, Karapetyan A, Lee W-J, Rugg E, et al.: Ovol2 Suppresses Cell Cycling and Terminal Differentiation of Keratinocytes by Directly Repressing C- Myc and Notch1. J Biol Chem 2009;284:29125-29135.

Wu RS, Hong JJ, Wu JF, Yan S, Wu D, Liu N, et al.: OVOL2 antagonizes TGF- $\beta$ signaling to regulate epithelial to mesenchymal transition during mammary tumor metastasis. Oncotarget 2017; DOI: 10.18632/oncotarget.17031

Xie C, Liu G, Li M, Fang Y, Qian K, Tang Y, et al.: Targeting TRPV1 on cellular plasticity regulated by Ovol 2 and Zeb 1 in hepatocellular carcinoma. Biomed Pharmacother 2019; DOI: 10.1016/j.biopha.2019.109270

Xu C, Yan T, Yang J: OVOL1 inhibits oral squamous cell carcinoma growth and metastasis by suppressing zinc finger E-box binding homeobox 1. Int J Clin Exp Pathol 2019;1:2801-2808.

Ye GD, Sun G Bin, Jiao P, Chen C, Liu QF, Huang XL, et al.: OVOL2, an Inhibitor of WNT Signaling, Reduces Invasive Activities of Human and Mouse Cancer Cells and Is Downregulated in Human Colorectal Tumors. Gastroenterology 2016; DOI: 10.1053/j.gastro.2015.11.041

Zhang T, Zhu Q, Xie Z, Chen Y, Qiao Y, Li L, et al.: The zinc finger transcription factor Ovol2 acts downstream of the bone morphogenetic protein pathway to regulate the cell fate decision between neuroectoderm and mesendoderm. J Biol Chem 2013; DOI: 10.1074/jbc.M112.418376

Zhou JX, Huang S: Understanding gene circuits at cell-fate branch points for rational cell reprogramming. Trends Genet 2011;27:55-62.

\section{Figure Legends}

Fig. 1: Specific and overlapping functions of OVOL1/2. OVOL proteins are crucial regulators of epithelial differentiation - they can inhibit EMT and induce MET across contexts.

Fig. 2: OVOL/ZEB1 axis correlates with EMT/MET across cancer types. (Left) Scatter plot of genes correlated using Pearson's correlation with OVOL1 and ZEB1 in CCLE dataset. Each dot denotes one gene and the $x$ - and $y$-axis coordinates are correlation coefficient values of that gene with OVOL1 and ZEB1 respectively. Color of the dots represents corresponding pvalues. Green dots are for genes with $p<0.05$ with OVOL1 and ZEB1. (Right) Same as the left scatter plot but for OVOL2 and OVOL1.

Fig. 3: OVOL/ZEB1 axis regulating EMT/MET in various biological contexts. OVOL and ZEB1 drive opposite (epithelial vs. mesenchymal respectively) differentiation in both normal epithelial differentiation as well as in cancer metastasis. 